\title{
Synovial chondromatosis in unusual locations treated with arthroscopy: A report of three cases
}

\author{
Mustafa Odluyurt ${ }^{1}\left(\mathbb{D}\right.$, Özlem Orhan ${ }^{2}$ (D), Erdem Aras Sezgin ${ }^{3}\left(\mathbb{D}\right.$, Ulunay Kanatı ${ }^{4}(\mathbb{D}$ \\ 'Department of Orthopedics and Traumatology, Zonguldak Çaycuma State Hospital, Zonguldak, Turkey \\ ${ }^{2}$ Department of Orthopedics and Traumatology, Harran University Faculty of Medicine, Şanlıurfa, Turkey \\ ${ }^{3}$ Department of Orthopedics and Traumatology, Aksaray University Faculty of Medicine, Aksaray, Turkey \\ ${ }^{4}$ Department of Orthopedics and Traumatology, Gazi University Faculty of Medicine, Ankara, Turkey
}

Synovium holds proliferative properties which may in some cases result in abnormal production of small clumps or nodules of hyaline cartilage. These nodules eventually break free from synovial tissue in time and become loose bodies with varying sizes in the joint. While smaller bodies lead to locking symptoms in the joint, larger ones cause mechanical erosion of the cartilage. Knee is the most commonly affected joint followed by hip. ${ }^{[1]}$ There are also reports about wrist, elbow and, far less commonly, shoulder and ankle joint involvement. ${ }^{[2-4]}$ The classical approach would be arthrotomy and synovectomy, but owing to advancements in arthroscopic techniques and instrument design, arthroscopy has emerged as a viable option in recent years. ${ }^{[3,4]}$ Although arthroscopy is a much less invasive technique with faster recovery

Received: November 06, 2021

Accepted: January 05, 2022

Published online: February 10, 2022

Correspondence: Özlem Orhan, MD. Harran Üniversitesi Tıp Fakültesi Ortopedi ve Travmatoloji Anabilim Dalı, 63300 Şanlıurfa, Türkiye.

E-mail: droorhan@gmail.com

Doi: 10.52312/jdrscr.2022.1

Citation: Odluyurt M, Orhan Ö, Sezgin EA, Kanatı U. Synovial chondromatosis in unusual locations treated with arthroscopy: A report of three cases. Jt Dis Relat Surg Case Rep 2022;1(2):63-66.

(02022 All right reserved by the Turkish Joint Diseases Foundation

This is an open access article under the terms of the Creative Commons Attribution-NonCommercial License, which permits use, distribution and reproduction in any medium, provided the original work is properly cited and is not used for commercial purposes (http://creativecommons.org/licenses/by-nc/4.0/).

https://www.casereportsjointdrs.org

\section{ABSTRACT}

Synovial chondromatosis (SC) rarely occurs in the shoulder and ankle joints. An intervention is necessary, as irreversible cartilage injury or transformation to chondrosarcoma may occur. Offering advantages such as faster recovery and lower rates of complication, arthroscopic techniques can be considered instead of open surgery. Herein, we describe three cases of SC, one in shoulder and two in ankle joints. Patients were young adult males and all underwent arthroscopic excision and two had synovectomy. At 18 months of follow-up there was no recurrence and no malignancy and symptoms were alleviated in all patients. In conclusion, arthroscopic removal of all loose bodies and synovectomy in primary SC can be regarded as a safe and effective treatment with low morbidity, rapid recovery, and good outcomes. Histological confirmation of the diagnosis is necessary as malignant transformation is possible.

Keywords: Ankle, arthroscopy, shoulder, synovectomy, synovial chondromatosis.

and lower morbidity, removing multiple loose bodies of varying sizes requires experience and patience.

In this article, we report three cases who underwent arthroscopic excision of multiple loose bodies from shoulder and ankle joints with the diagnosis of synovial chondromatosis (SC).

\section{CASE REPORT}

All three cases were informed that data from the case would be submitted for publication and gave their consent.

Case 1- A 19-year-old male student with no history of chronic disease was admitted with right shoulder pain for one year, which was exacerbated while putting on clothes. Pain worsened within the past three months and was constant and often woke 


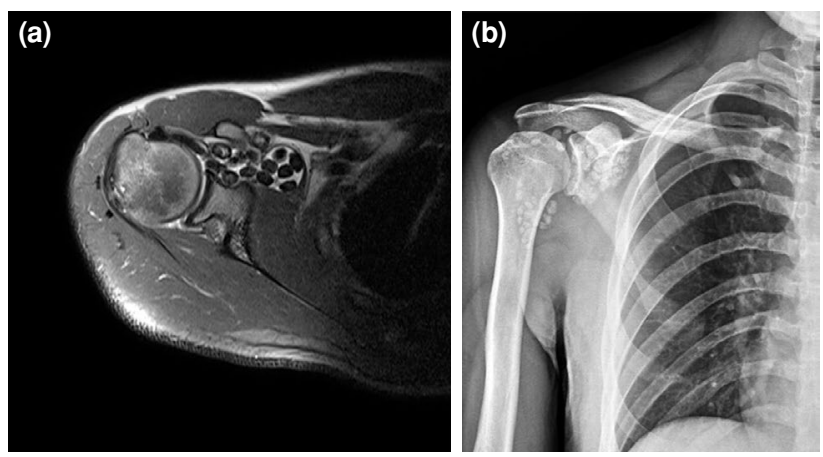

Figure 1. Preoperative radiographic and magnetic resonance images of the patient with shoulder synovial chondromatosis. (a) On proton-density-weighted transverse oblique magnetic resonance image of the shoulder, nodular hypointense signals localized in the subscapularis recess and subcoracoid bursa. (b) Anteroposterior radiograph of the shoulder shows many loose bodies extensively located in the bicipital groove and in the subcoracoid recess.

him up from sleep. Physical examination showed limited range of motion and tenderness. Flexion, extension, abduction, internal rotation, and external rotation were limited to $110^{\circ}, 30^{\circ}, 100^{\circ}, 30^{\circ}$ and $20^{\circ}$, respectively. A grinding sensation was felt during palpation. Radiographic images of the shoulder and magnetic resonance imaging (MRI) revealed multiple calcific nodules in the glenohumeral joint and degenerative changes (Figure 1). During arthroscopy utilizing a standard posterior portal, multiple loose bodies in the axillary pouch and subcoracoid space, rotator interval, fraying of the humeral head cartilage, fraying of the long head of biceps tendon was observed (Figure 2a). Active synovial budding was observed (Figure $2 \mathrm{~b}$ ). Following removal of 50 cartilaginous loose bodies interchanging portals, motorized shaver was used to perform partial synovectomy (Figure 2c, d). Intraoperative radiographic images showed no loose bodies and increased passive range of motion was observed. The patient was discharged $48 \mathrm{~h}$ after the operation and early range of motion exercises were immediately encouraged. Pathological examination of loose bodies confirmed SC (Figure 3). Postoperative two-year follow-up showed relief of symptoms and activity increased closer to the previous levels. Flexion, extension, abduction, internal rotation, and external rotation were increased to $170^{\circ}, 50^{\circ}, 160^{\circ}, 70^{\circ}$ and $40^{\circ}$, respectively.

Case 2- A 24-year-old, otherwise healthy male, was admitted to our outpatient clinic with anterior right ankle pain associated with activity for six months. He had a limp, and his examination revealed a mild effusion and limited ankle dorsiflexion $\left(5^{\circ}\right)$. Radiographs and MRI revealed a calcified mass localized intra-articularly on the anterior aspect of the ankle joint (Figure 4). After conservative treatment failed, the calcified mass which was $15 \times 8 \mathrm{~mm}$ in size was excised with arthroscopy (Figure 5). Follow-up at postoperative 30 months showed complete relief of symptoms and increased dorsiflexion $\left(20^{\circ}\right)$.

Case 3- A 27-year-old male, without history of trauma and other diseases, having pain, tenderness, grinding sensation and limited range of motion of the left ankle for a year, was admitted to our outpatient clinic. Dorsiflexion, plantar flexion, eversion, and inversion were limited to $5^{\circ}, 30^{\circ}, 10^{\circ}$ and $15^{\circ}$, respectively. Radiological techniques revealed multiple calcified loose bodies located anteriorly in the left ankle joint. Standard anterior portals were used to arthroscopically excise multiple loose bodies (Figure 6). Similar to the first case presented in this report, owing to the proliferative activity in the synovium, partial synovectomy was performed with a motorized shaver. Tibiotalar joint degeneration was noted during arthroscopy. At 18-month follow-up, the
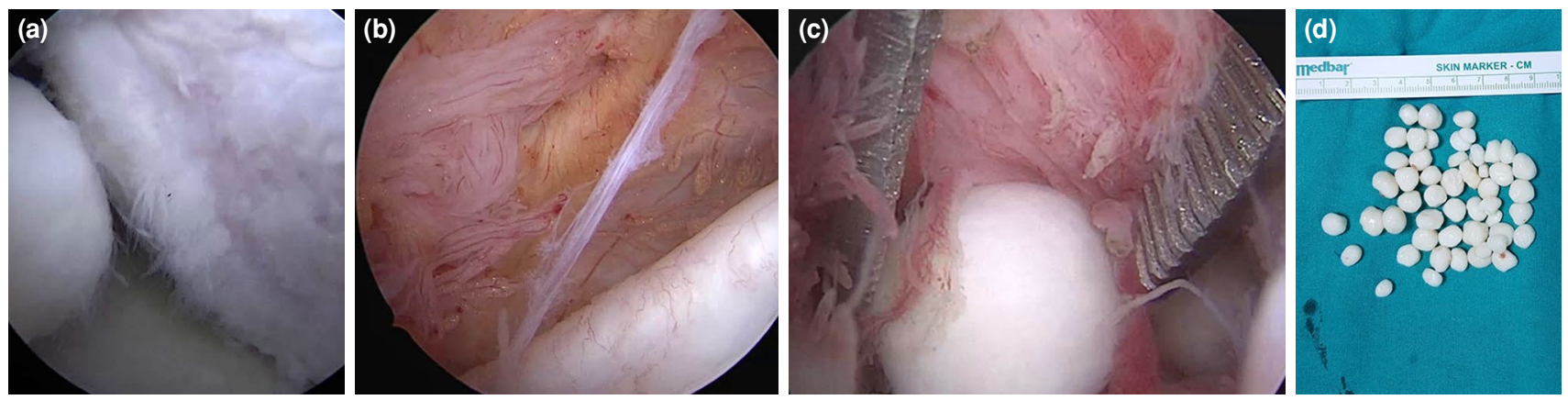

Figure 2. (a) Intraoperative arthroscopic images show fraying of the cartilage on the humeral head, (b) active synovial budding and inflammation and (c) removal of a large loose body with an artery forceps. (d) A total of 50 loose bodies with varying sizes were excised. 


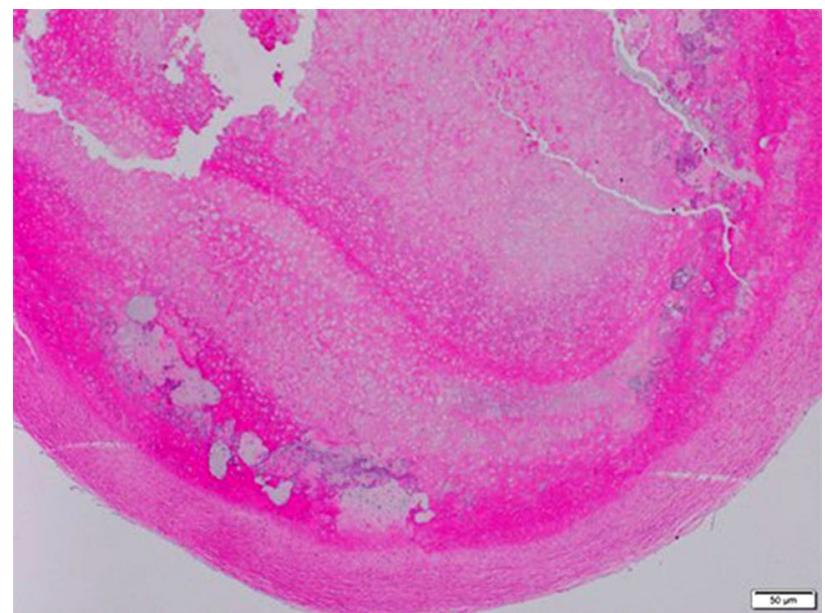

Figure 3. Hematoxylin and eosin-stained slide with $\times 20$ magnification shows cellular cartilage closer to the surface. patient had increased range of motion (dorsiflexion, plantar flexion, eversion and inversion were $15^{\circ}, 45^{\circ}$, $20^{\circ}$ and $25^{\circ}$, respectively) and had mostly alleviated symptoms.

\section{DISCUSSION}

Primary SC usually has an insidious progression which can result in delayed diagnosis. Severe irreversible articular cartilage lesions of articular surfaces may be apparent upon admission, as in our patient with shoulder involvement. Computed tomography (CT) or MRI are usually necessary to aid in defining the exact localization of the disease. The latter has the advantage of identifying non-calcified bodies on the initial phase of the disease. Younger lesions can be identified with their isointense signal
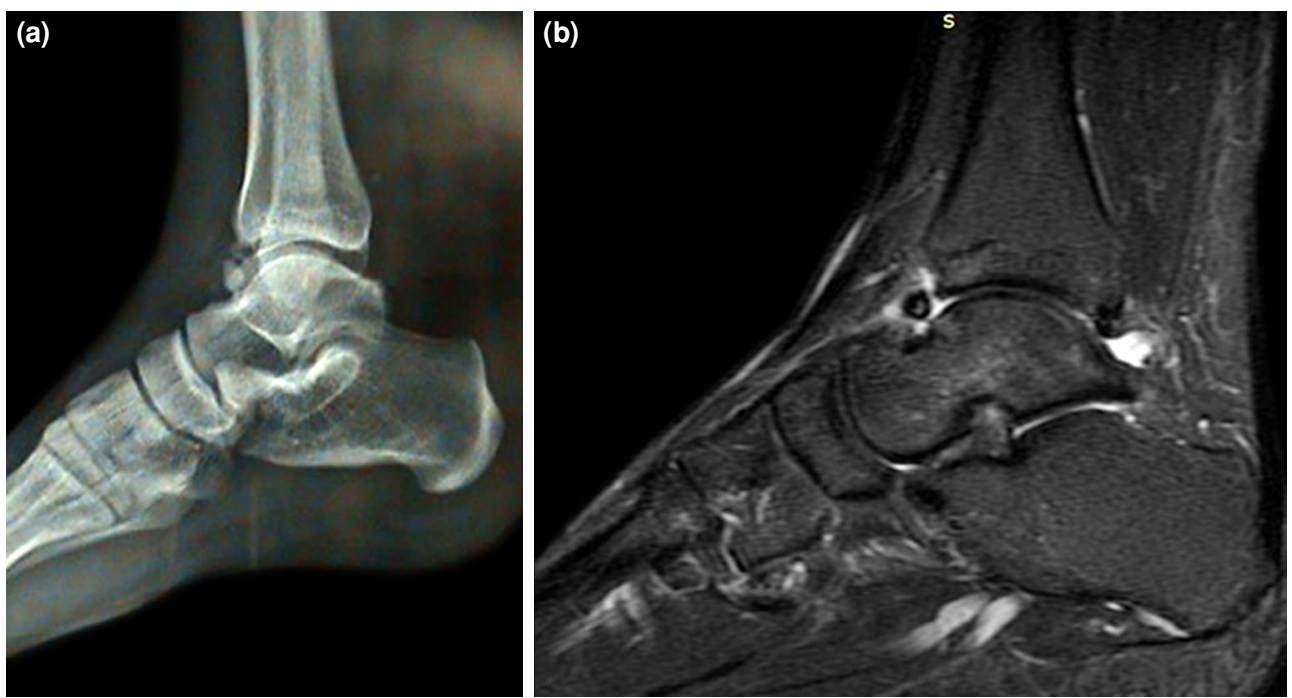

Figure 4. (a) Preoperative lateral ankle radiograph and (b) T2-weighted sagittal magnetic resonance image of the second patient show a single nodule located in the anterior compartment of the ankle.
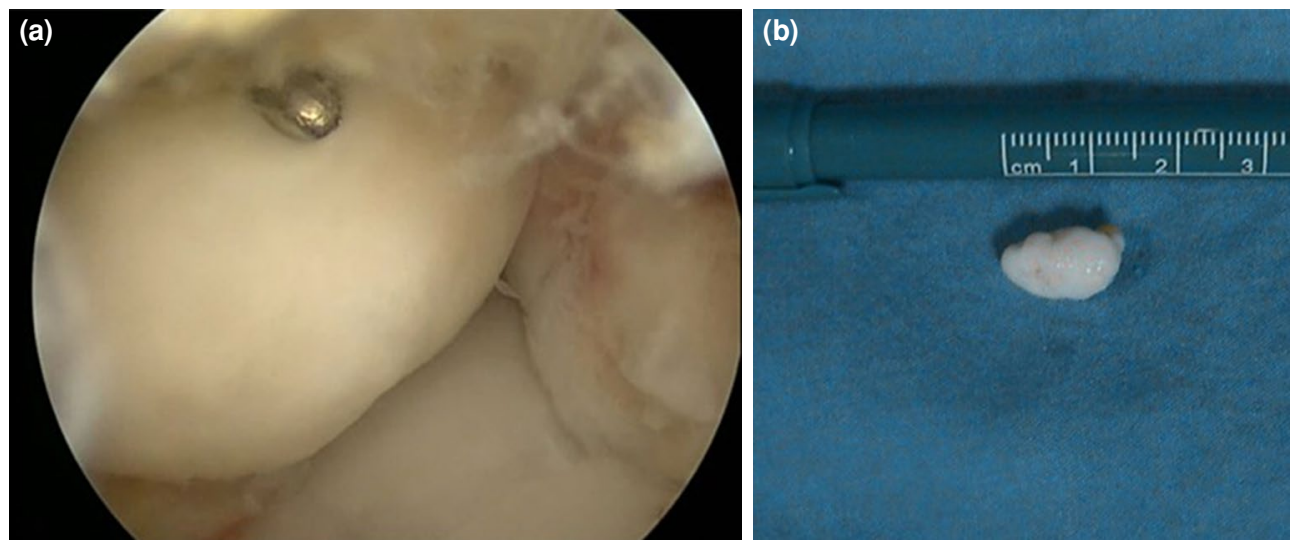

Figure 5. (a) An intraoperative arthroscopic image shows a large loose body located in the anterior compartment of the ankle. (b) The nodule had irregular borders and was $1.5 \times 0.8 \mathrm{~cm}$ in size. 


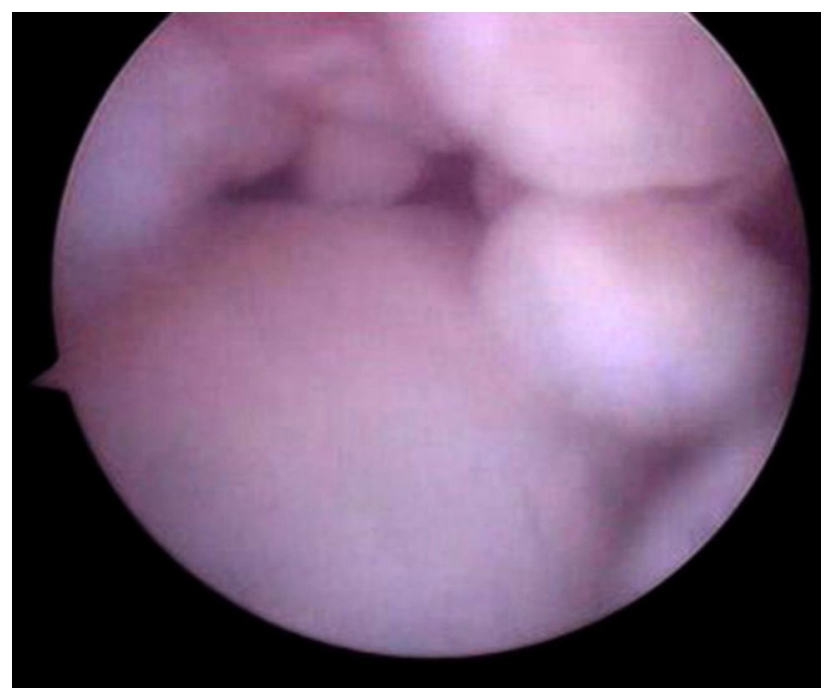

Figure 6. An intraoperative arthroscopic image shows multiple loose bodies in anterior compartment of the ankle joint.

on T1-weighted images, while calcifications appear as hypointense areas both in T1- and T2-weighted images. Synovial chondrosarcoma should always be kept in mind, while evaluating the differential diagnoses. Secondary SC, intra-articular chondroma, localized or general tenosynovial giant cell tumors are additional diagnoses to consider, particularly in the knee joint.

Three types of the disease were defined by Milgram $^{[5]}$ in 1977 combining radiographic and histopathological features: 1 or early stage, active budding without intra-articular loose bodies; 2 or intermediate stage, loose bodies with ongoing active budding in synovium; 3 or late stage, loose bodies without active synovial disease. These stages have been suggested to be important in making the treatment choice and estimating the outcome. Cases 1 and 3 in this report were on intermediate stage and, therefore, we decided to perform partial synovectomy, unlike the Case 2 who had late-stage disease.

Contemporary case reports and series report that partial synovectomy combined with removal of loose bodies performed by arthroscopy can provide significant relief and increased range of motion. Wahab et al. ${ }^{[6]}$ arthroscopically removed more than 120 loose bodies from the shoulder of a 20 -year-old man and performed partial synovectomy. Although there were significant degenerative changes, as in our case, the patient was pain free at two years. A similar case reported by Memon et al. ${ }^{[3]}$ also showed that arthroscopic excision of loose bodies followed by partial synovectomy and intra-articular methylprednisolone injection yielded good short- term results. In a very recent case series on 17 ankle SC cases, Bojanić et al. ${ }^{[4]}$ suggested that SC should be considered a whole joint disorder and should be treated with extensive synovectomy, combining anterior and posterior compartment ankle arthroscopy, even if loose bodies were limited to a single compartment. It is still not clear whether total synovectomy is mandatory. With the lack of long-term studies comparing these two treatment modalities, a definite conclusion cannot be made currently.

It should be kept in mind that malignant transformation is possible in up to $5 \%$ of cases. ${ }^{[7,8]}$ Recognizing the malignant process may be difficult and histological confirmation is crucial for establishing the definite diagnosis and lesion characterization.

In conclusion, our experience with these cases shows that arthroscopic removal of all loose bodies and synovectomy in primary SC can be regarded as a safe and effective treatment with low morbidity, rapid recovery, and good outcomes. However, arthroscopic expertise is necessary to reach all loose bodies and perform adequate synovectomy.

\section{Declaration of conflicting interests}

The authors declared no conflicts of interest with respect to the authorship and/or publication of this article.

\section{Funding}

The authors received no financial support for the research and/or authorship of this article.

\section{REFERENCES}

1. Neumann JA, Garrigues GE, Brigman BE, Eward WC. Synovial chondromatosis. JBJS Rev 2016;4:e2.

2. Saxena A, St Louis M. Synovial chondromatosis of the ankle: Report of two cases with 23 and 126 loose bodies. J Foot Ankle Surg 2017;56:182-6.

3. Memon F, Pawar ED, Gupta D, Yadav AK. Diagnosis and arthroscopic treatment of synovial chondromatosis of glenohumeral joint: A case report. J Orthop Case Rep 2021;11:59-62.

4. Bojanić I, Plečko M, Mataić A, Dimnjaković D. Anterior and posterior arthroscopic treatment of primary synovial chondromatosis of the ankle. Foot Ankle Int 2021;42:440-7.

5. Milgram JW. Synovial osteochondromatosis: A histopathological study of thirty cases. J Bone Joint Surg [Am] 1977;59:792-801.

6. Wahab H, Hasan O, Habib A, Baloch N. Arthroscopic removal of loose bodies in synovial chondromatosis of shoulder joint, unusual location of rare disease: A case report and literature review. Ann Med Surg (Lond) 2018;37:25-9.

7. Evans S, Boffano M, Chaudhry S, Jeys L, Grimer R. Synovial chondrosarcoma arising in synovial chondromatosis. Sarcoma 2014;2014:647939.

8. Biazzo A, Confalonieri N. Synovial chondrosarcoma. Ann Transl Med 2016;4:280. 